\title{
Treatment of pulmonary aspergilloma with itraconazole
}

\author{
J H Campbell, J H Winter, M D Richardson, G S Shankland, S W Banham
}

\begin{abstract}
In a 12 month open study of itraconazole in pulmonary aspergilloma nine patients received oral itraconazole $200 \mathrm{mg}$ daily for six months followed by further itraconazole or observation for a further six months. There was no change in the serum IgG specific for Aspergillus fumigatus (mean (SE) change - 4\% $(10 \%)$ ) or symptoms of chronic cough and haemoptysis. In two of the three patients who continued treatment beyond six months symptoms and radiographic appearances improved and a temporary reduction in $A$ fumigatus specific IgG occurred in one patient. Further experience of the effects of longer treatment are needed before oral itraconazole can be recommended for aspergilloma.
\end{abstract}

Pulmonary aspergillomas are a recognised complication of cavitating lung disease, occurring in $10-15 \%$ of patients. ${ }^{12}$ Although many patients are symptomless at presentation, aspergillomas have an unpredictable natural history and are a substantial additional burden in terms of morbidity and mortality, ${ }^{3}$ life threatening haemoptysis and the development of invasive aspergillosis being the most serious complications. At present there is no consensus on the most appropriate treatment and this is reflected in the variety of treatments used. Many of these patients have serious underlying lung disease that precludes surgery and the development of effective oral antifungal treatment would be particularly useful for this group.

Itraconazole is an orally active triazole antifungal agent with less toxicity and greater in vitro activity against Aspergillus fumigatus than other available agents. ${ }^{4}$ These features make it a potentially attractive treatment for pulmonary aspergilloma. We conducted an open study of oral itraconazole in nine patients with aspergilloma to determine the effects of treatment on symptoms, radiographic appearances, and the antibody response to Aspergillus.

\section{Methods}

Symptomatic patients aged over 18 years with radiologically defined aspergilloma and IgG antibodies to $A$ fumigatus were eligible irrespective of the underlying lung disease. Nine patients were entered into the study (table). Four patients had a history of at least one major haemoptysis and all had chronic cough or intermittent haemoptysis or both.

All patients received oral itraconazole $200 \mathrm{mg}$ capsules daily for six months. Their response was determined each month on the basis of symptoms, clinical signs, and levels of IgG antibody to $A$ fumigatus. Chest radiography and lung function tests were performed every two months, with tomography at entry and at six months. In addition, patients kept a diary for 14 days of each month recording symptoms of cough, wheeze, and haemoptysis on a digital scale. After six months the investigators had the option of continuing itraconazole or of observation for a further six months.

Serum IgG specific for $A$ fumigatus was determined by a rapid enzyme linked immunosorbent assay (ELISA). ${ }^{5-7}$ This was standardised by including samples of precipitin positive and negative reference serum in each assay and by expressing results as percentages of a positive control to provide a single unit of activity. ${ }^{6}$ This approach overcame intertest variation and permitted comparison between assays.

Serum precipitins specific for $A$ fumigatus were determined by counterimmunoelectrophoresis. ${ }^{8}$ For each sample a titre was obtained by testing doubling dilutions of the patient's serum against two concentrations of $A$ fumigatus cytoplasmic antigen.

\section{Results}

\section{RADIOLOGY}

Two patients showed a minor reduction in size of their aspergilloma after six months' treatment. Both patients continued treatment for a further six months-by which time in one patient (No 4) the aspergilloma had disappeared completely (fig 1) and in the other (No 2) there had been a substantial reduction in size and rounding of its contour. The aspergilloma recurred in patient 4 within three months of her stopping itraconazole. In the other seven patients (six of whom received treatment for six months only) there was no radiological change.

\section{ANTIBODIES TO A FUMIGATUS}

The coefficient of variation between replicate samples of individual serum specimens within each assay was always less than $10 \%$. Variation between the positive and the negative internal standard panels of serum was minimal. The same reference serum samples were used throughout the study. Variation between patients in the level of $A$ fumigatus specific IgG 
Details of the patients and their aspergillomas

\begin{tabular}{|c|c|c|c|c|c|}
\hline \multirow[b]{2}{*}{$\begin{array}{l}\text { Patient } \\
\text { No }\end{array}$} & \multirow[b]{2}{*}{$\begin{array}{l}\text { Age } \\
(y)\end{array}$} & \multirow[b]{2}{*}{ Sex } & \multirow[b]{2}{*}{$\begin{array}{l}\text { Underlying lung } \\
\text { disease }\end{array}$} & \multicolumn{2}{|c|}{ Aspergilloma } \\
\hline & & & & $\begin{array}{l}\text { Duration } \\
(y)\end{array}$ & $\begin{array}{l}\text { Site } \\
\text { (radiological) }\end{array}$ \\
\hline 1 & 59 & $M$ & Tuberculosis & 7 & $\begin{array}{l}\text { Right middle lobe, } \\
\text { previous lobectomy }\end{array}$ \\
\hline 2 & 62 & $\mathbf{M}$ & Tuberculosis & 6 & Right upper lobe \\
\hline 3 & 57 & $\mathbf{F}$ & Tuberculosis & 8 & Right apex, thick walled \\
\hline 4 & 58 & $\mathbf{F}$ & Tuberculosis & 8 & Right upper lobe \\
\hline 5 & 59 & $\mathbf{M}$ & Tuberculosis & 6 & Right apex \\
\hline 6 & 61 & $\mathbf{F}$ & Pneumonia & 6 & Right upper lobe \\
\hline 7 & 55 & $\mathbf{F}$ & Tuberculosis & 5 & Upper lobe, bilateral fibrosis \\
\hline 8 & 53 & $\mathbf{M}$ & Tuberculosis & 7 & $\begin{array}{l}\text { Right upper lobe, } \\
\text { bilateral bronchiectasis }\end{array}$ \\
\hline 9 & 51 & $\mathbf{F}$ & Sarcoidosis & 8 & $\begin{array}{l}\text { Left apex, bilateral } \\
\text { fibrosis of upper lobe }\end{array}$ \\
\hline
\end{tabular}

was considerable (fig 2). There was also a wide range of precipitin titres between patients at each time of measurement during the study, though the levels of IgG and precipitin titre for individual patients were in general agreement.

There was no significant change in specific IgG (fig 2) or precipitins to $A$ fumigatus for the group during the six months of treatment. The

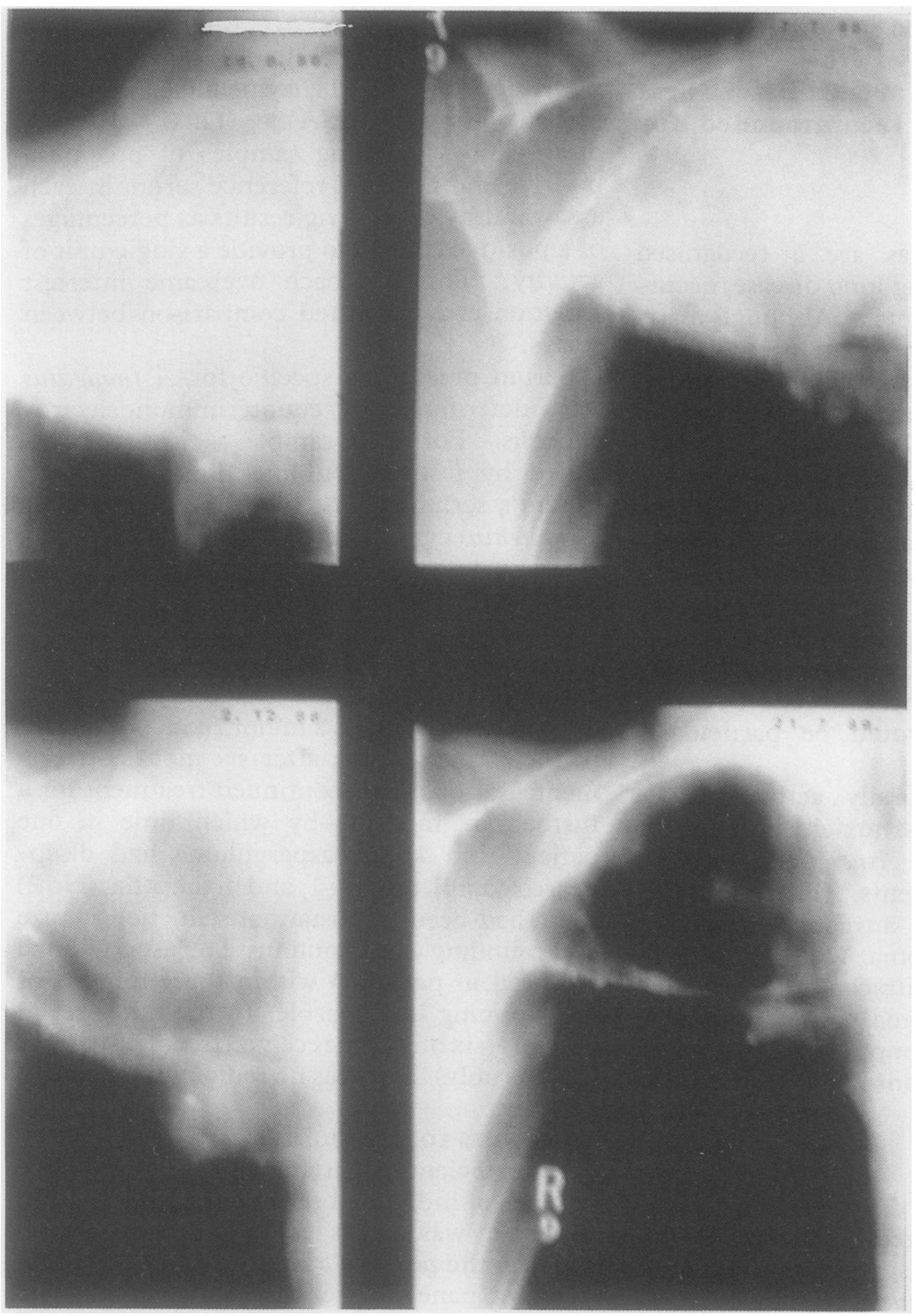

Figure 1 Serial chest radiographs for patient 4, showing gradual disappearance of right upper lobe aspergilloma over 12 months (at entry and two, six, and 12 months later). radiological response in patient 4 was associated with a $50 \%$ reduction in specific IgG levels but the antibody level rose and symptoms returned within two months of her stopping itraconazole.

\section{SPIROMETRY}

Treatment produced no change in either the FEV $_{1}$ (before: mean 1.25 (SE 0.21) l; after: 1.25 $(0.29) 1)$ or the forced vital capacity (before: $1.86(0.25)$ l; after: $1.92(0.31) 1)$.

\section{CLINICAL ASPECTS}

One patient (No 8) had a major haemoptysis while having the treatment, which settled without further intervention. In the two patients with a radiological response haemoptysis stopped and in one of them the anaemia and high erythrocyte sedimentation rate were corrected.

Itraconazole was well tolerated. One patient developed unstable angina one month after starting treatment and underwent successful coronary artery bypass grafting. The relevance of the angina is uncertain. There was no change in serum transaminase, bilirubin, or blood count during the six months.

DEATHS

Two patients died during the follow up period after stopping itraconazole. One, aged 55 years and with extensive post-tuberculous upper lobe fibrosis, died from respiratory failure after infection a month after stopping itraconazole; the other, aged 51 years with inactive pulmonary sarcoidosis and a left apical aspergilloma, died after a massive haemoptysis seven months after stopping treatment. Neither had shown any signs of response to itraconazole.

\section{Discussion}

The best approach to the patient with an aspergilloma is not clear. Treatment is aimed at reducing the complications of massive haemoptysis and invasive aspergillosis. For many years resection has been accepted as the best option in those fit for surgery. Medical management with intravenous or percutaneous intracavitary antifungal agents, embolisation of bronchial arteries, radiotherapy, and cavernoscopic treatment have met with varying success. $^{9-12}$ Until the 1970 s antifungal treatment was restricted because of unacceptable side effects, but the advent of the azole group of antifungal agents brought the possibility of effective oral treatment. Shale and coworkers ${ }^{13}$ found lower levels of serum IgG antibody to $A$ fumigatus and less severe symptoms in patients with allergic bronchopulmonary aspergillosis or aspergilloma treated with oral ketoconazole than in those given placebo. Three of their patients had aspergilloma, of whom two received ketoconazole, with resolution of the aspergilloma in one (though the aspergilloma diminished in size in the patient who took placebo). Ketoconazole has been associated with hepatitis and its suitability for long term treatment in this condition must be questioned. Itraconazole has potential advantages in that 


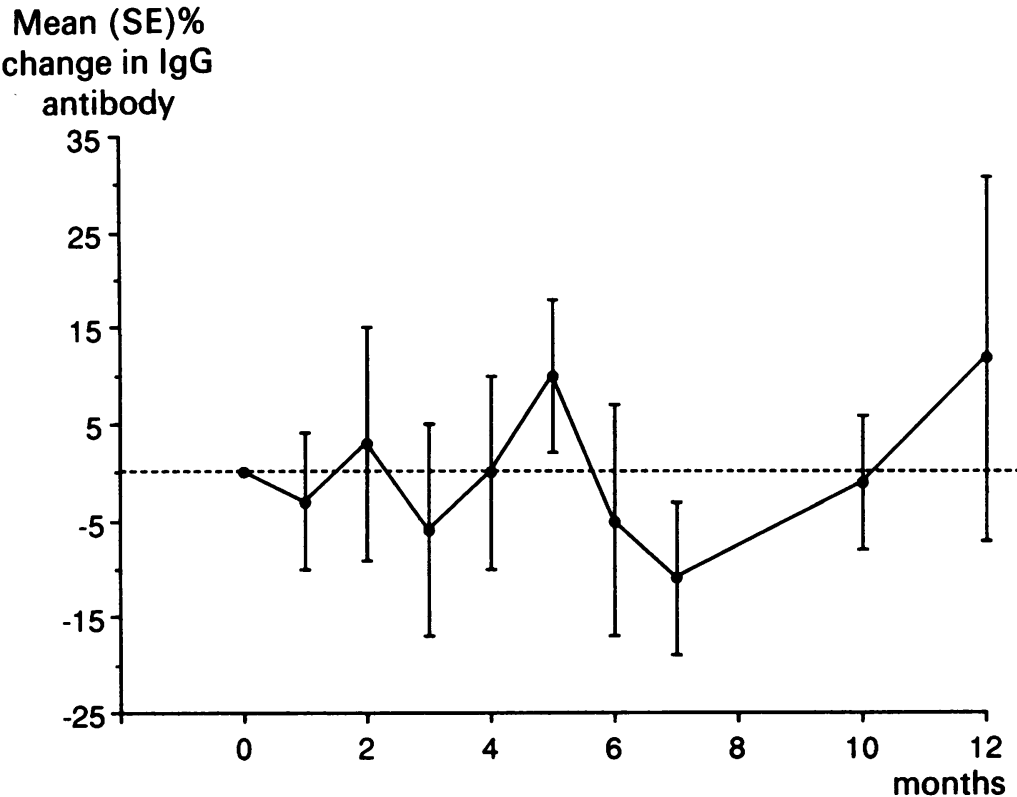

Figure 2 Effects of itraconazole on levels of IgG antibody to Aspergillus fumigatus expressed as mean change from baseline and standard error of mean.

hepatic damage has not been reported and it has greater in vitro activity against $A$ fumigatus. In a group of patients with various diseases related to Aspergillus, including aspergilloma, oral itraconazole treatment was associated with symptomatic improvement in 21 of 34 patients evaluated and radiological improvement in 12 of 40 patients (though only one case resolved completely) after they had taken itraconazole for a variable period. ${ }^{14}$

The lack of consistent immunological or symptomatic improvement in our patients is therefore disappointing. The immunological response to antifungal treatment is complex and possibly treatment might increase the immunological response initially, especially if it caused only partial damage to the fungal ball. Despite essentially negative findings several points arise from this study. Of the three patients who continued itraconazole for a year, two had a late response, and in these patients the radiological, symptomatic, and immunological responses were impressive. This late response provides some support for the view that prolonged (11-15 months') antifungal treatment is required for an effect on the chronic forms of aspergillosis, ${ }^{15}$ though spontaneous lysis of aspergillomas may occur in up to $10 \%$ of cases. ${ }^{316}$ This unpredictable natural history makes it difficult to attribute change to a specific treatment, though most spontaneous regressions reported have occurred within 36 months of diagnosis. Both our responders had a much longer history (eight years) and one of these patients had a recurrence of the aspergilloma within three months of stopping itraconazole. This supports the view that the aspergilloma had responded to itraconazole.

Itraconazole cannot at present be recommended for standard treatment of aspergilloma but the possibility of a late response when itraconazole is taken for more than six months warrants further study.

1 Macpherson P. Pulmonary aspergillosis in Argyll. $\mathrm{Br} J \mathrm{Dis}$ Chest 1965;59:148-57.

2 British Tuberculosis and Thoracic Association. Aspergillus in persistent lung cavities after tuberculosis. Tubercle 1968;49:1-11.

3 Jewkes J, Kay PH, Paneth M, Citron KM. Pulmonary aspergilloma: analysis of prognosis in relation to haemoptysis and survey of treatment. Thorax 1983;38:572-8.

4 Van Cutsem J, Van Gerven F, Van De Ven MA, Borgers M, Janssen PAJ. Itraconazole, a new triazole that is orally active in aspergillosis. Antimicrob Agent Chemother active in aspergi

5 Richardson MD, Stubbins JM, Warnock DW. Rapid enzyme-linked immunosorbent assay (ELISA) for Aspergillus fumigatus antibodies. J Clin Pathol 1982;35:1134-7.

6 Richardson MD, Turner A, Warnock DW, Llewellyn PA Computer-assisted rapid enzyme-linked immunosorbent assay (ELISA) in the serological diagnosis of aspergillosis. assay (ELISA) in the serological diagn

7 Richardson MD. Mycology. In: Wreghitt TG, MorganCapner $\mathrm{P}$, eds. ELISA in the clinical microbiology laboratory. London: Public Health Laboratory Service, 1990:238-60.

8 Mackenzie DWR. Serological tests. In: Evans EGV, Richardson MD, eds. Medical mycology: a practical approach. Oxford: Oxford University Press, 1989:201-33.

9 Hughes CF, Waugh R, Lindsay D. Surgery for pulmonary aspergilloma: pre-operative embolisation of the bronchial circulation. Thorax 1986;41:324-5.

10 Shapiro MJ, Albedla SM, Mayock RL, McLean GK. Severe haemoptysis associated with pulmonary aspergilloma: precutaneous intracavitary treatment. Chest 1988;94: precutaneous 1225 - 1 .

11 Shneerson JM, Emerson PA, Philips RH. Radiotherapy for massive haemoptysis from an aspergilloma. Thorax 1980; 35:953-4.

12 Subba R, Curzon PGD, Muers MF, Watson DA. Cavernoscopic evacuation of aspergilloma: an alternative method of palliation for haemoptysis in high risk patients. Thorax 1984;39:394-6.

13 Shale DJ, Faux JA, Lane DJ. Trial of ketoconazole in noninvasive pulmonary aspergillosis. Thorax 1987;42:26-31.

14 De Beule K, De Doncker P, Cauwenbergh G, et al. The treatment of aspergillosis and aspergilloma with itraconazole, clinical results of an open international study (19821987). Mycosis 1988;31:476-85.

15 Restrepo A, Munera MI, Arteaga D, Gomez I, Tabares AM, Arango M. Developments in the treatment of aspergilloma [abstract]. In: Topics in mycology aspergullus and aspergillosis: 1987: Second symposium. 99-101.

16 Hammerman KJ, Christianson CS, Huntington I, Hurst GA, Zelman M, Tosh FE. Spontaneous lysis of aspergillomata. Chest 1973;64:697-9. 